\title{
A Multi-agent System for Emergency Decision Support
}

\author{
Martin Molina, Gemma Blasco \\ Department of Artificial Intelligence, Universidad Politécnica de Madrid, \\ Campus de Montegancedo S/N 28660 Boadilla del Monte, Madrid, Spain \\ mmolina@fi.upm.es, gblasco@isys.dia.fi.upm.es
}

\begin{abstract}
This paper describes the multi-agent organization of a computer system that was designed to assist operators in decision making in the presence of emergencies. The application was developed for the case of emergencies caused by river floods. It operates on real-time receiving data recorded by sensors (rainfall, water levels, flows, etc.) and applies multi-agent techniques to interpret the data, predict the future behavior and recommend control actions. The system includes an advanced knowledge based architecture with multiple symbolic representation with uncertainty models (bayesian networks). This system has been applied and validated at two particular sites in Spain (the Jucar basin and the South basin).
\end{abstract}

\section{Introduction}

In Spain, the SAIH National Programme (Spanish acronym for Automatic System Information in Hydrology) was initiated with the goal of installing sensor devices and telecommunications networks in the main river basins to get on real time in a control center the information on rainfall, water levels and flows in river channels. One of the main goals of this type of control centers is to help to react in the presence emergency situations as a consequence of river floods.

This task may be facilitated with the help of a computer system conceived as an intelligent assistant [1]. The development of such a system presents certain difficulties such as: (1) the system has to include a model to automatically provide on real time answers about future hypotheses of behavior, (2) the system has to explain and justify its conclusions given that the operator must take the final responsibility of decisions, and (3), since the SAIH Programme develops several of realizations of such systems for different river basins, it is also important to consider the reusability of the software architecture.

In this paper we describe the multi-agent organization of a decision support computer system, called SAIDA, for flood emergency management in the context of the SAIH Programme. The paper describes first the functional model, describing the main tasks: evaluation, prediction and recommendation. Then, the paper describes how these tasks are simulated in a distributed manner according to a multiagent architecture. Finally, the last section presents how the system was implemented with the help of a knowledge engineering tool. 


\section{The functional model for decision support}

In order to develop the SAIDA system, we formulated a knowledge model based on the tasks that operators perform during flood emergency situations. For each task we selected an appropriate problem solving method that help to construct the computational version [2]. According to this, the resulting knowledge model for SAIDA includes three main tasks: evaluation, prediction and recommendation. The goal of the evaluation task is to identify potential emergency situations by interpreting sensor data about the current state of the river basin, based on certain patterns of scenarios about rain, water levels, flows and reservoir states. This corresponds to a typical classification task that selects a category (a type of problem) within a prefixed set, based on a set of observations (sensor data). In this case, this task can be solved by an adaptation of the heuristic classification method [3] with two steps: (1) abstract to abstract data from sensors, and (2) match to find patterns of problems that match the current information from sensors.

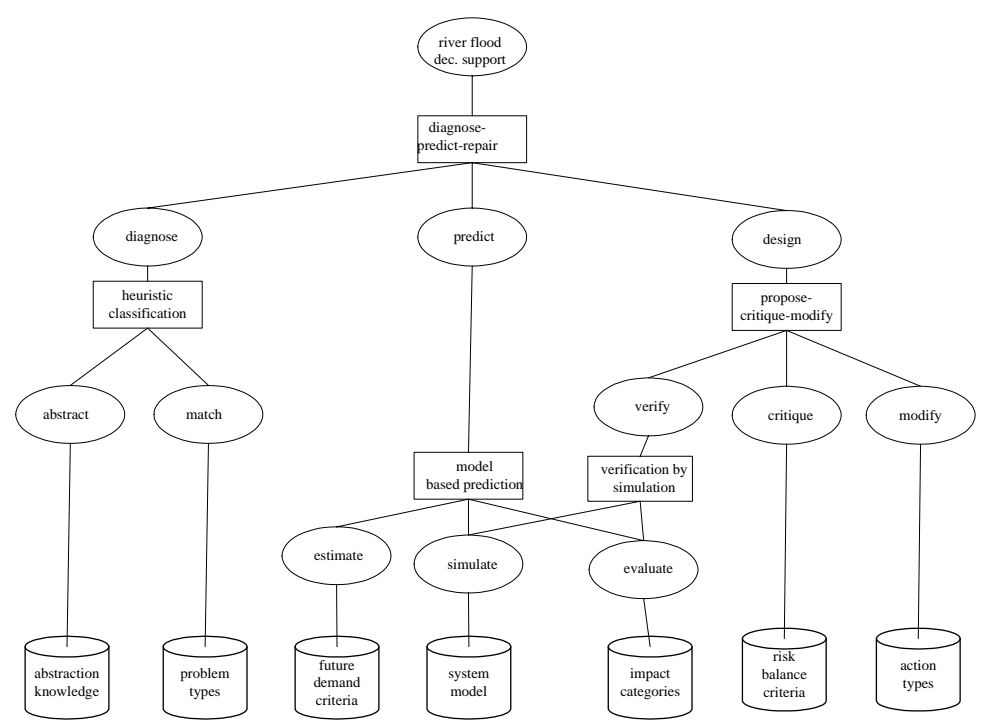

Figure 1: A functional view of the model for decision support in the field of emergency management during floods. Legend: circle (task), square (method), cylinder (knowledge base).

The goal of the prediction task is to predict the future behavior and estimate potential damages. This task can be carried out by a method that performs the following steps: (1) estimate future rain to generate hypotheses of future rain for the next $\mathrm{H}$ hours (e.g. $\mathrm{H}=8$ hours), based on a heuristic model about the river basin and the global weather forecast received as input, (2) simulate the river behavior that uses a model of the river basin based on causal relations following a bayesian approach in order to provide the range of potential behaviors using statistical measures, and (3) estimate potential damages to estimate the impact of the flows in terms of potential damages by using empirical knowledge that relates water levels and flows with qualitative ranges of severity for each particular critical location. 
The goal of the recommendation task is to suggest possible control actions as an answer to the detected problem. This distinguishes between two possibilities: (a) hydraulic actions, that establish discharge policies at the dams to avoid undesirable impacts and (b) defensive actions, such as population alert, evacuation procedures, etc. involving different organizations like traffic police, health services, fire brigades, army, etc. The first case can be performed by a method that explores a search space of potential hydraulic actions using a heuristic approach. The basic idea is that the method evaluates the current situation and, based on empirical knowledge, proposes a set of hydraulic actions that potentially can solve the problem. Then, these actions are tested by simulation and, if the result of the test is not satisfactory, empirical knowledge is used again to modify the hydraulic actions. These steps are performed in a loop until a satisfactory set of control actions is found. In the artificial intelligence literature, this method receives the name of propose-critique-modify [4][5]. The set of defensive actions is found by using a classification method supported by a model that relates types of problems with types of defensive actions (for the shake of simplicity, this method is not included in the figure corresponding to the complete model).

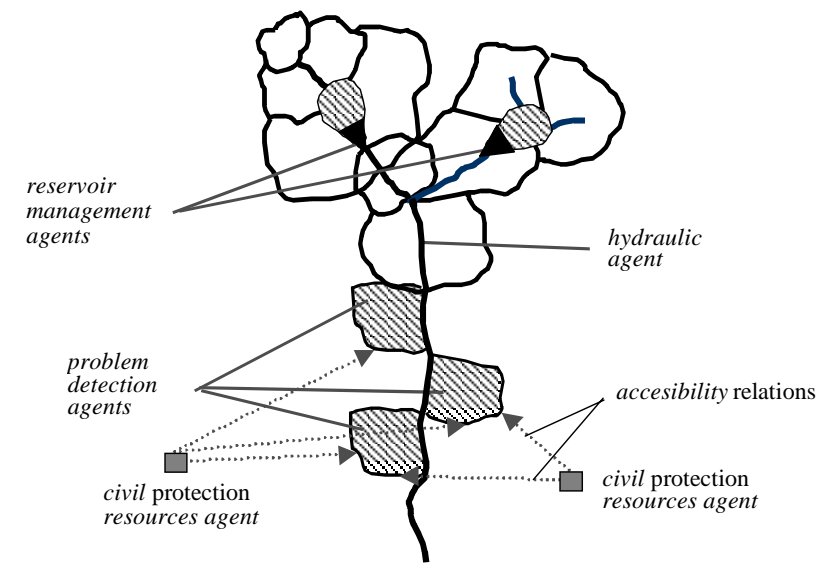

Figure 2: Types of agents

\section{The multi-agent organization}

In order to cope with the knowledge complexity, we followed the concept of multiagent system to complement the previous knowledge model. To develop the multiagent approach we followed the distributed nature of the decisions and the geographical distribution. We identified four types of agents: (1) hydraulic agents to give answers about the behavior of the physical process, (2) problem detection agents, to evaluate the flood risk in a particular geographical area, (3) reservoir management agents, with criteria for exploitation strategy for each reservoir, and (4) civil protection agents, responsible to provide with resources of different types according to the demands of the problem detection agents. 


\subsection{Domain knowledge distribution between agents}

Figure 3 shows how we distributed the different knowledge bases between agents. There is an agent for each specific decision point at certain location in the river basin depending on its nature (problem area, reservoir, river channel or protection). For each agent, there is a set of types of knowledge bases, each one with its particular language representation. For example, problem detection agents include a total of six knowledge bases that provide the necessary domain criteria for the agent's tasks. Some of the types of knowledge bases for problem detection agents are the same for reservoir management agents. They have the same representation and role in the reasoning process but the content is different for each agent.

A model for a particular river basin is constructed formulating this set of knowledge bases. Figure 3 shows a summary of a complete model for the case of a river basin in Spain (the Júcar river basin). This includes a total of 23 agents, one for each specific decision point at certain location in the river basin depending on its nature (problem area, reservoir, river channel or protection). For each agent, there is a set of types of knowledge bases, each one with its particular language representation, with a total of 143 knowledge bases.

\begin{tabular}{|c|c|c|c|c|}
\hline Agents & $\begin{array}{l}\text { N. of } \\
\text { agents }\end{array}$ & $\begin{array}{l}\text { Knowledge } \\
\text { Bases }\end{array}$ & $\begin{array}{l}\text { Knowledge } \\
\text { Representation }\end{array}$ & $\begin{array}{l}\text { N. of } \\
\text { KBs }\end{array}$ \\
\hline \multirow{6}{*}{$\begin{array}{l}\text { Problem } \\
\text { detection agents }\end{array}$} & \multirow[t]{6}{*}{15} & Abstraction & Functional + temporal represent. & 15 \\
\hline & & Problem types & Frames with uncertainty degrees & 15 \\
\hline & & Future Demand & Rules & 15 \\
\hline & & Impact categories & Bayesian network & 15 \\
\hline & & Risk balance criteria & Rules & 1 \\
\hline & & Action types: agent relations & Horn Logic Clauses & 15 \\
\hline \multirow{6}{*}{$\begin{array}{l}\text { Reservoir } \\
\text { management } \\
\text { agents }\end{array}$} & \multirow[t]{6}{*}{4} & Abstraction & Functional + temporal represent. & 4 \\
\hline & & Problem types & Frames with uncertainty degrees & 4 \\
\hline & & Future Demand & Rules & 4 \\
\hline & & Impact categories & Bayesian nets & 4 \\
\hline & & Risk balance criteria & Rules & 1 \\
\hline & & Action types: discharge strategies & Rules & 1 \\
\hline \multirow{6}{*}{$\begin{array}{l}\text { Hydraulic } \\
\text { agents }\end{array}$} & \multirow[t]{6}{*}{2} & Abstraction & Functional + temporal represent. & 2 \\
\hline & & System model: influence diagram & Temporal causal network & 2 \\
\hline & & System model: infiltration & Bayesian network & 12 \\
\hline & & System model: discharge & Bayesian network & 12 \\
\hline & & System model: reservoir discharge & Bayesian network & 4 \\
\hline & & System model: junction & Bayesian network & 11 \\
\hline \multirow{3}{*}{$\begin{array}{l}\text { Protection } \\
\text { agents }\end{array}$} & \multirow[t]{3}{*}{2} & Action types: transport network & Rules & 2 \\
\hline & & Action types: population & Rules & 2 \\
\hline & & Action types: constructions & Rules & 2 \\
\hline TOTAL & $23 \mathrm{Ag}$. & & TOTAL & $143 \mathrm{KBs}$ \\
\hline
\end{tabular}

Figure 3: Summary of knowledge bases corresponding to a particular model

\subsection{Task distribution between agents}

According to the multi-agent organization, the previous three tasks (evaluation, prediction and recommendation) are distributed among the different agents, in such a way that they communicate partial results to complete general goals. For example, the 
evaluation task is distributed among problem detection agents, in such a way that each one includes local knowledge to determine whether in its local geographical area there is a significant risk of floods according to the current state of the river basin. For this purpose they consult data from sensors and classify the situation according to the method for evaluation described in the previous section.

When a particular problem detection agent identifies a potential dangerous scenario, it asks for a prediction of behavior to the corresponding hydraulic agents and, then, it interprets this information to conclude the level of severity of the future problem. In order to predict the behavior of the river, the hydraulic agent is specialized in simulating the local physical phenomena (rainfall, runoff, etc.) of a particular river, by using local river models based on bayesian networks.

The last of the three tasks (the recommendation task) is carried out by a method that simulates how the agents with potential problems ask other agents to help them in decreasing their risk level by performing a kind of cooperation. For this purpose, we designed a multi-agent version of the centralized knowledge-based method called propose-critique-modify. Based on this method, we consider the following steps (figure 4): (1) propose, a new state of the river basin is proposed by simulation (using bayesian networks) considering the effect of a current control action, (2) critique the current proposal of hydraulic actions, (2) modify the current proposal of hydraulic action based on the previous critiques and following certain heuristic rules.

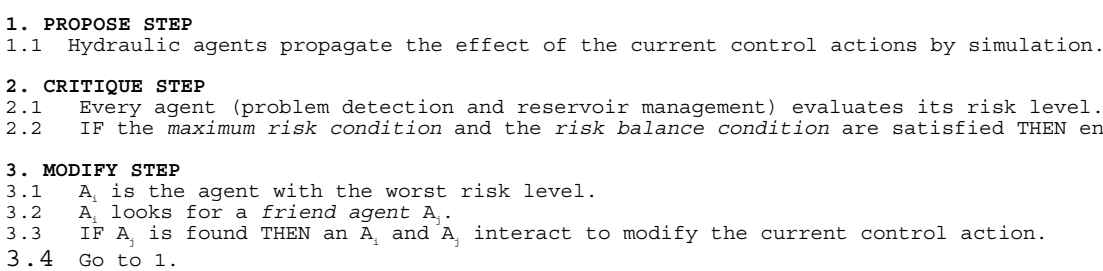

Figure 4: Multi-agent version of the propose-critique-modify method

In order to perform the critique step, the model uses the concept of risk level. The risk level is a discrete value that goes from 1 to 10 and represents globally the severity of the situation at certain location. For the case of problem detection agents the risk level is evaluated based on the sort-term prediction of the flow in the problem area. For this purpose, a bayesian network is defined for each location in such a way that low (high) values of flow are related with low (high) values of risk. The bayesian representation provides the required freedom to calibrate each particular case according to the specific physical structure. The risk level is computed for every future time step so that the final risk level is the maximum value of the values of risk level for each time step. For the case of reservoir management agents the risk level is computed based on the sort-term prediction of the reservoir storage. For this purpose a parameter $S$ (safe factor) is computed with $\mathrm{S}=100\left(\mathrm{C}-\mathrm{V}_{0}\right) /\left(\mathrm{V}_{\max }-\mathrm{V}_{0}\right)$ where $\mathrm{C}$ is the capacity of the reservoir, $\mathrm{V}_{0}$ is the current storage of the reservoir and $\mathrm{V}_{\max }$ is the maximum storage corresponding to the sort-term prediction. Then, the risk level is determined using conditional heuristic relations in form of production rules in such a 
way that low levels of safe factor and high values of current storage are related with high values of risk levels.

Taking into account that the goal is to minimize the maximum risk level, the critique step uses a social rule expressed with two conditions that determines when the current hydraulic control is acceptable:

- Maximum risk condition: Every agent must present a risk level lower than a threshold (e.g., lower than 5).

- Risk balance condition: The different between the highest and lowest risk levels must be lower than a threshold (e.g., lower than 3).

During the modify step, the goal is to find a new proposal of control actions by considering how to decrease the highest risk level. This step considers the social rule that the agent $A_{i}$ with the highest risk level is in charge of finding a new proposal. Since the reduction of a risk level for a particular agent usually implies to increase the risk level of others, $A_{i}$ looks for another agent $A_{j}$, called the friend agent, that could help by increasing its own risk level. $A_{j}$ is considered a friend of $A_{i}$ when:

- $A_{i}$ is a reservoir management agent, $A_{j}$ is a downstream agent of $A_{i}$, there are not river confluences between $A_{i}$ and $A_{j}, A_{j}$ presents a low risk level, or

- $A_{i}$ is an agent (reservoir management or problem detection), $A_{j}$ is an upstream reservoir management agent, $A_{j}$ is not closed, there are not closed reservoirs between $A_{i}$ and $A_{j}, A_{j}$ presents a low risk level.

If the friend agent is found, an interaction between both agents is performed based on local heuristic rules to find a control action. For instance, this interaction is based on the idea that an agent $A_{i}$ (e.g., problem detection agent) asks to the other $A_{j}$ (e.g., reservoir agent) to decrease the discharge in order to decrease its own risk level one point. For this purpose $A_{i}$ computes locally the desired risk level to determine the flow decrease and $A_{j}$ computes locally the flow decrease to determine the new storage objective. As a result of the interaction, one of the agents, $A_{i}$ or $A_{j}$, proposes a modification of the control in form of a new storage objective for a particular reservoir. Then, the effect of this control action is simulated and the process continues in an iterative loop until a satisfactory solution is found.

\section{Implementation}

To implement the SAIDA system we used a knowledge engineering tool called KSM [6]. With this tool it is possible to formulate an abstract knowledge model as a structured collection of knowledge bases and problem-solvers, supported by a library of primitives of representation (software components that implement each type of knowledge representation and inference). Then, this abstract structure can be instantiated in different ways, using specific domain knowledge, to produce different knowledge systems. 


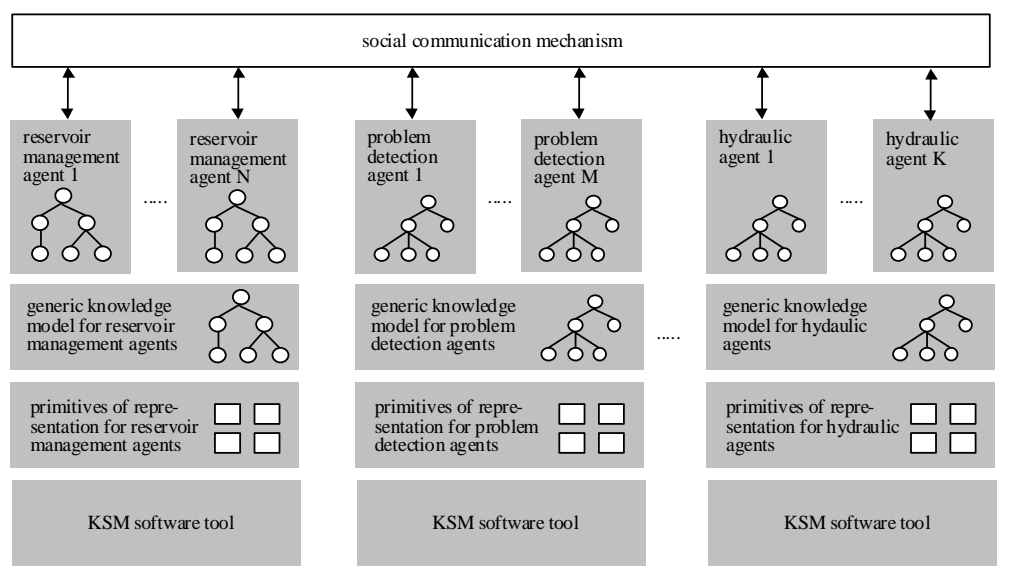

Figure 5: Software architecture to support the multi-agent organization.

We followed this idea to define an abstract knowledge organization for each type of agent (reservoir management agent, problem detection agent, etc.) as a structured collection of classes of tasks, knowledge bases, symbolic representation, etc. Then, each specific agent (e.g., the Forata reservoir management agent) is built as particularization of the abstract structure providing domain knowledge (specific domain rules, etc.)[7]. Thus, different copies of KSM, one for each family with the same generic structure, support the complete society of agents (Figure 5). Therefore, KSM provided a solution to build general knowledge structures that are shared by different instances of agents, giving the required freedom to write the particular adaptations of each agent in their particular knowledge bases.

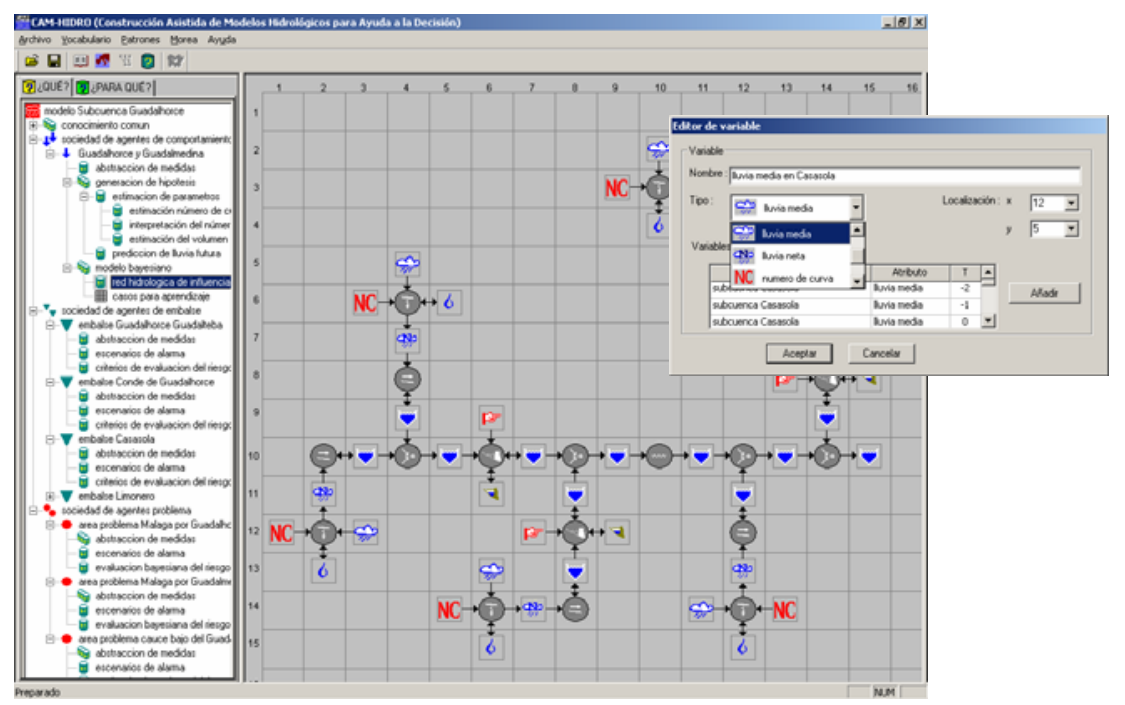

Figure 6: Example of screen presented by the user interface of the CAM-Hidro tool. 
On the other hand, to facilitate the model construction and maintenance for each particular river basin, we built a software tool called CAM-Hidro that assists developers and end-users providing guidance and assistance for consistency checking and complementary views (abstract views, dependence relations, etc.) of the model.

\section{Conclusions}

In summary, the application presented in this paper, SAIDA, corresponds to an innovative multi-agent based solution for a system with complex and heterogeneous agents. Each type of agent was designed as a knowledge-based system with multiple representation (rules, bayesian networks, logic clauses, etc.) and different tasks. The multi-agent approach was very useful to organize in a modular and natural structure the different components of the whole knowledge model.

We developed two realizations of this system for two different river basins in Spain. For instance, the Júcar River Basin included 23 agents with a total of 143 knowledge bases. For the implementation, the KSM tool provided enough flexibility and efficiency to operate on real time and the CAM-Hidro tool were useful to facilitate the process of construction and maintenance of knowledge models.

Acknowledgements. The development of the SAIDA system was supported by the Ministry of Environment of Spain (Dir. General de Obras Hidráulicas y Calidad de las Aguas) with the participation of local public organizations from river basins (Conf. Hidrográfica del Júcar and Conf. Hidrográfica del Sur de España.). The development of the CAM-Hidro tool was supported by the Ministry of Science and Technology of Spain within the RIADA project.

\section{References}

1. Boy, G., Gruber T.R.: "Intelligent Assistant Systems: Support for Integrated HumanMachine Systems” Proc. of 1990 AAAI Spring Symposium on Knowledge-Based HumanComputer Communication, March 1990, Stanford University.

2. Molina M., Ossowski S.: "Knowledge Modelling in Multiagent Systems: The Case of the Management of a National Network" in "Intelligence in Service and Networks. Paving the Way for an Open Service Market" Lecture Notes in Computer Science 1597, Springer, 1999.

3. W. Clancey: "Heuristic Classification”. Artificial Intelligence 27, 1985.

4. Brown D., Chandrasekaran B.: Design Problem-solving: Knowledge Structures and Control Strategies, Morgan Kaufman, 1989.

5. Marcus S., McDermott J.: "SALT: A knowledge acquisition language for propose-andrevise systems”. Artificial Intelligence, 39(1) 1-38, 1989.

6. J. Cuena, M. Molina: "The Role of Knowledge Modeling Techniques in Software Development: A General Approach Based on a Knowledge Management Tool". International Journal of Human-Computer Studies (2000) 52, 385-421.

7. Molina M., Cuena J.: Using Knowledge Modelling Tools for Agent-based Systems: The Experience of KSM. In J.Cuena et al. (eds.) "Knowledge Engineering and Agent Technology” IOS Press, Ámsterdam, in press (2003). 